\title{
Performance Analysis of Crosstalk Subcarrier Multiplexing and Wave Division Multiplexing in Optical Communication System
}

Ebrahim E. Elsayed ( $\nabla$ engebrahem16@gmail.com)

Mansoura University Faculty of Engineering https://orcid.org/0000-0002-7208-2194

Research Article

Keywords: WDM, BER, OBI, SCM, SNR, SMF

Posted Date: June 24th, 2021

DOI: https://doi.org/10.21203/rs.3.rs-460310/v2

License: (c) (1) This work is licensed under a Creative Commons Attribution 4.0 International License.

Read Full License 


\title{
Performance Analysis of Crosstalk Subcarrier Multiplexing and Wave Division Multiplexing in Optical Communication System
}

\author{
Ebrahim E. Elsayed \\ Electronics and Communications Engineering Department, Faculty of Engineering, \\ Mansoura University, Mansoura 35516, El-Dakahilia Governorate, Egypt.
}

Author's email address: engebrahem16@gmail.com

\begin{abstract}
In traditional optical communication, duplexity is achieved by using two fibers, each having a transmitter and a receiver. Economically, bidirectional wavelength division multiplexing (WDM) transmission systems utilizing a single fiber will be more attractive not only reducing the use of the fiber by a factor of two ,but also the number of components. Duplex transmissions over a single fiber can double the capacity of an installed unidirectional link. The idea of this paper is to study another approach using the subcarrier multiplexing (SCM)-based optical network and evaluate the physical transmission quality of analog and digital signal using SCM approach and the characteristic of fiber nonlinear crosstalk such as stimulated Raman scattering, Cross phase modulation and four-wave mixing in the SCM externally modulation optical link. A suitable bandwidth of $890-950 \mathrm{MHz}$ is selected for subcarriers and channel bandwidth of $200 \mathrm{KHz}$ and carrier. By measuring the optical bit interference (OBI) performance limitations of the subcarrier multiplexing WDM optical transmission system is investigated. The OBI for 10 channels for input power $1 \mathrm{~dB}$ is $-40 \mathrm{~dB}$ whereas for 110 channels the $\mathrm{OBI}$ is $-20 \mathrm{~dB}$ separation of 250 $\mathrm{KHz}$ are considered.
\end{abstract}

Keywords: WDM, BER, OBI, SCM, SNR, SMF.

\section{1) INTRODUCTION}

An optical communications system is similar to other communication systems in that it consists of the three main parts: Transmitter, Receiver and the Communication channel [1-3]. In order for a fiber to guide the light signal, it must consist of a core of material whose refractive index is greater than that of the surrounding medium, which is called the cladding. Depending on the design of the fiber, light is constrained to the core by either total internal reflection or refraction [4].

\section{2) The Basic Blocks of Fiber Optical Transmission Link}

The basic block of an optical fiber transmission system is illustrated in Figure 1 consists of three main parts: The transmitter block "Laser Driver and temperature control" [5-8]: the electrical signals will be transferred into optics. For long haul, laser diode is used for this purpose because of the narrow spectral 
width and high optical power that is used to carry data over long distance. The light is then coupled into the transmission channel, the optical fiber cable, where most of the dispersion and attenuation takes place [5]. The receiver block which is the last part of the system converts the optical signal back into the replica of the electrical signal using the Avalanche photodiode (APD) or PIN-type photodiode then to the amplification stage before reaching the end user [9-12].

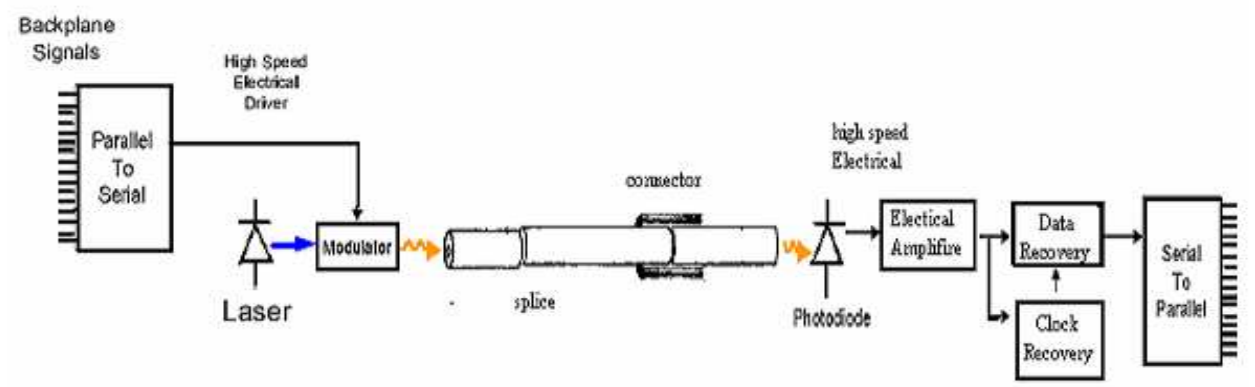

Figure 1: Basic block diagram of fiber optic communication.

3) Wavelength Division Multiplexing (WDM) System

WDM as shown in Figure 2, $[1,13-16]$ combines multiple optical TDM data streams onto one fiber through the use of multiple wavelengths of light. Each individual TDM data stream is sent over an individual laser transmitting a unique wavelength of light. Wavelength division multiplexing was used with only two wavelengths $1310 \mathrm{~nm}$ and $1550 \mathrm{~nm}$. However, this was suitable only for limited applications for example; applications in which analog optical cable television signals co-existed with digital optical telecommunication signals. WDM takes advantage of the fact that different wavelengths of light can be transmitted over a single fiber simultaneously $[17,18]$.

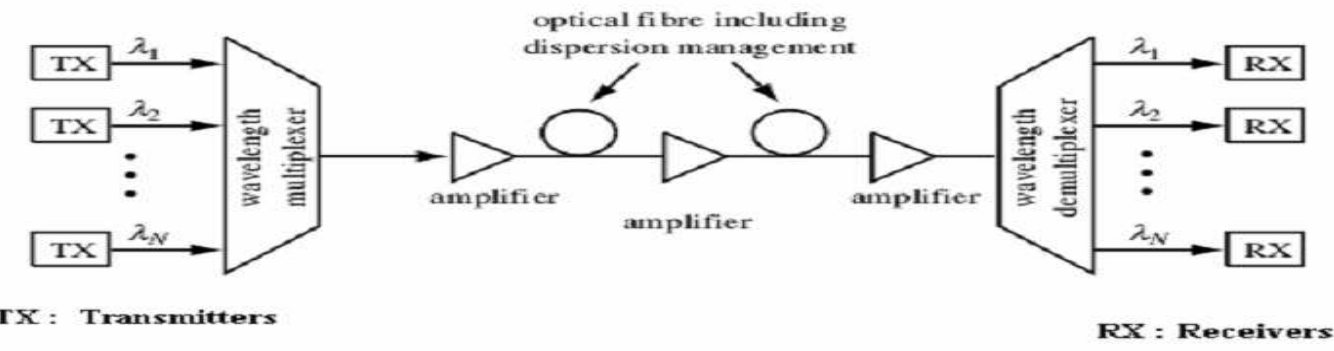

Figure 2: Schematic representation of the wavelength division multiplexing system. 


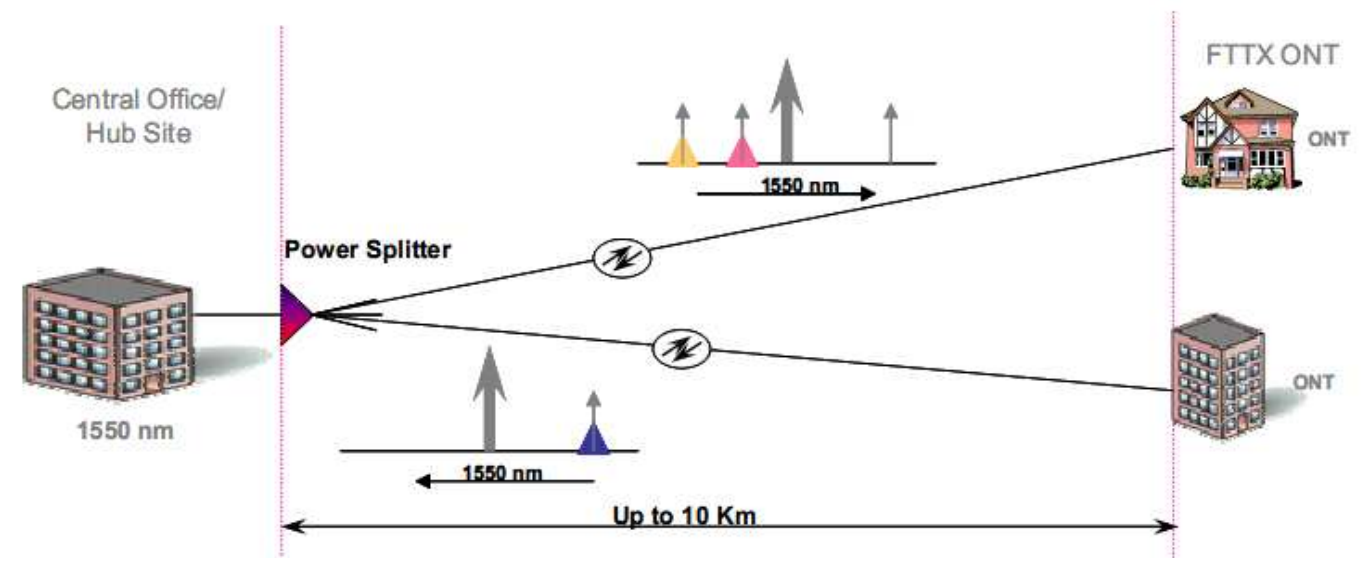

Figure 3: SCM/WDM architecture.

\section{4) Types of Crosstalk}

- Crosstalk due to Filtering [15-19]

* Crosstalk element superimposed on the signal as a random power adding process. However, there is no significant beating element. A narrow band optical filter would largely reduce the electrical impairment [18-20].

- Linear Crosstalk

\section{Space switches crosstalk.}

Homo wavelength crosstalk.

Hetero wavelength crosstalk.

- Non-linear Crosstalk

Four wave mixing (FWM) : Wave mixing to give rise of new frequency

* Cross phase modulation: Intensity dependent refractive index.

Scattering: Transfer of power between propagation modes.

\section{5) Fiber Nonlinear Crosstalk}

In addition to transmitter and receiver noises in optical systems, fiber nonlinear crosstalk can significantly degrade the transmission system performance. There are two basic fiber nonlinear mechanisms [1, 4, and 15]. The first mechanism that causes fiber nonlinearities is the scattering phenomena, which produces Stimulated Raman Scattering. The second mechanism arises from the refractive index of glass being dependent on the optical power going through the material. This results in producing Cross Phase Modulation (XPM) and FWM crosstalk [13-16]. 
- Stimulated Raman Scattering (SRS) Crosstalk Frequency Response. Stimulated Raman Scattering (SRS) is a nonlinear phenomenon found in wavelength-division multiplexed (WDM) transmission system. As shown in figure 3, where the shorter wavelength channels are robbed of power and that power feeds the longer wavelength channel [9, 2026].

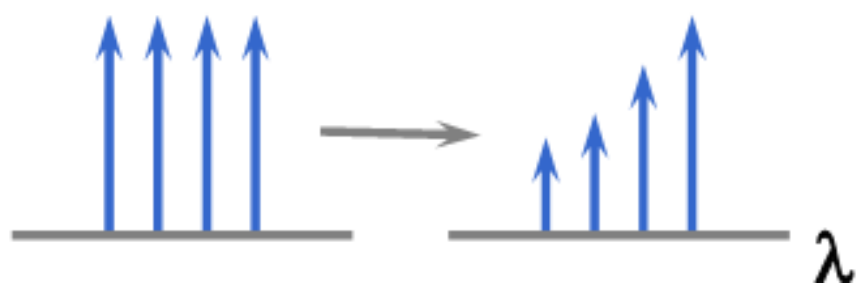

- Figure 4: Stimulated Raman scattering (SRS) crosstalk frequency response.

As for the crosstalk interaction between pump channel and signal channel in the SCM/WDM system, assuming pump channel has a shorter wavelength than probe channel, the most significant crosstalk term is due to the SRS interaction between pump channel optical carrier and probe channel subcarriers [18-20, 26-30].

A formal approach to determining SRS crosstalk levels is to solve the coupled propagation equations for the optical intensity I at wavelengths $\lambda 1$ and $\lambda 2[10,15]$.

$$
\begin{aligned}
& \frac{\partial I_{1}}{\partial z}+\frac{I}{v_{1}} \frac{\partial I_{1}}{\partial t}=\left(g I_{2}-\alpha\right) I_{1} E q .1 \\
& \frac{\partial I_{2}}{\partial z}+\frac{I}{v_{2}} \frac{\partial I_{2}}{\partial t}=\left(-g I_{1}-\alpha\right) I_{2} E q .2
\end{aligned}
$$

Where $\mathrm{z}$ is the distance along the fiber, $\mathrm{g}$ is the Raman gain (loss) coefficient and $v$ is the group velocity of each channel in the fiber. Assuming $\lambda 2<\lambda 1$ (channel 2 is designated as pump channel and channel 1 as probe channel). By neglecting the SRS term, $g I_{2}$ on the right hand side Eq. 1 of and solving for $I_{1}$; then substituting $I_{1}$ into Eq.2 and solving for $I_{2}$ are gets $[10,15]$ :

$$
P_{2}\left(0, \mu_{2}\right)=P_{2}\left(0, \mu_{2}\right) e^{-\alpha z} \cdot \exp \left[-\frac{g}{A_{e f f}} \int_{0}^{z} P_{1}\left(0, \mu_{2}+d_{12} z^{\prime}\right) e^{-\alpha z^{\prime}} d z^{\prime}\right] E q .3
$$




$$
P_{2}\left(0, \mu_{2}\right) \approx P_{2}\left(0, \mu_{2}\right) e^{-\alpha z} \cdot\left[1-\frac{g}{A_{e f f}} \int_{0}^{z} P_{1}\left(0, \mu_{2}+d_{12} z^{\prime}\right) e^{-\alpha z^{\prime}} d z^{\prime}\right] \text { Eq. } 4
$$

Where $d_{12}=\left|\frac{1}{v_{1}}-\frac{1}{v_{2}}\right|$ is the group velocity mismatch between the pump and signal channels and $\mu_{2}=t-\frac{z}{v_{2}}$

A similar approach can be used to solve for $I_{1}$ by neglecting the SRS term, $-g I_{1}$ on the right hand side of Eq.2 and solving for $I_{2}$, then substituting $I_{2}$ into Eq.1 to get [10, 15]

$$
P_{1}\left(0, \mu_{1}\right) \approx P_{1}\left(0, \mu_{1}\right) e^{-\alpha z} \cdot\left[1+\frac{g}{A_{e f f}} \int_{0}^{z} P_{2}\left(0, \mu_{1}+d_{12} z^{\prime}\right) e^{-\alpha z^{\prime}} d z^{\prime}\right] E q .5
$$

\section{6) Analysis of SCM in Presence of OBI}

There are $\mathrm{M}$ numbers of subcarrier multiplexing (SCM) in a given optical channel, having the same average power. Each of these fields can be represented by $[11,12]$ :

$$
e_{i}(t)=\sqrt{S_{i}(t)} \quad \text { Eq. } 6
$$

Where $S_{i}$ the intensity modulation by an RF is signal of center frequency $f_{i}$ and can be represented by [11-15]:

$$
S_{i}=1+m(t) \cos \left(2 \pi f_{i} t\right) E q .7
$$

Where $\mathrm{m}(\mathrm{t})$ is NRZ data signal with bit period $T_{b}$.

The total field in an optical channel is the sum of $\mathrm{M}$ fields and can be represented as $[11,20]$ :

$$
e_{i n}(t)=\sum_{i=1}^{M} e_{i}(t) \quad E q .8
$$

The electric field at the output of the fiber is given by:

$$
e_{o}(t)=\left|e_{i n}(t) * h_{f}(t)\right| e^{-\alpha L} E q .9
$$

Where $\alpha$ is the fiber attenuation coefficient, L is the fiber length and $h_{f}(t)$ represents the fiber impulse response.

The photodetector (PD) converts this field into an electrical signal proportional to the field intensity. 
$i_{s}(t)=\left\{\sum_{i=1}^{M} e_{o}^{2}(t)\right\}$

$i_{c}(t)=\left\{2 \sum_{i=1}^{M} \sum_{I=i+1}^{M} e_{i}(t) e_{1}(t)\right\}$

Here $\boldsymbol{i}_{\boldsymbol{c}}(\boldsymbol{t})$ contributes nonzero beat interference terms. The output of the PD is passed through a pre-amplifier followed by a band pass filter. If any of the spectral components of $\boldsymbol{i}_{\boldsymbol{c}}(\boldsymbol{t})$ falls within the bandwidth of any of the M users BPF, it will cause OBI.

$$
\begin{aligned}
i_{s}(i)=\int_{-\infty}^{+\infty} i_{s(i)}(t) e^{(-j f t)} & \\
= & 2 \pi \delta(f)+\left(\frac{T}{2}\right) \\
& *\left[\operatorname{sinc} T\left(f-f_{i}\right)+\operatorname{sinc} T\left(f+f_{i}\right)\right] e^{\left(-\frac{j f T}{2}\right)}
\end{aligned}
$$

Where $\boldsymbol{f}_{\boldsymbol{i}}$ represents the required subcarrier frequency.

The power spectrum of the i-th subscriber's signal component can be expressed as:

$$
\begin{aligned}
P_{s}(i)(f)=\left[i_{s}(i)(f)\right]^{2} & \\
& =4 \pi 2 \delta(f)+\left(\frac{T 2}{4}\right) \\
& *\left[\operatorname{sinc}^{2} T\left(f-f_{i}\right)+\operatorname{sinc}^{2} T\left(f+f_{i}\right)\right] e^{\left(-\frac{j f r}{2}\right)} \text { Eq. } 13
\end{aligned}
$$

Using band pass filter, output signal power of the required sub-carrier can be calculated as [11-15]:

$P i(\operatorname{sig})=\int_{f_{i}-\frac{B}{2}}^{f_{i}+\frac{B}{2}} P_{s(i)}(f) d f=\int_{f_{i}-\frac{B}{2}}^{f_{i}+\frac{B}{2}}\left(\frac{\tau 2}{4}\right) *[\operatorname{sinc} 2 \tau(f-f i)] d f \quad E q .14$

Where B is the specified bandwidth of the subcarrier or bandwidth of the BPF.

This $P_{i}$ (cross) is the source of the OBI.

SNR $=\frac{\boldsymbol{P}_{\boldsymbol{i}}(\boldsymbol{s i g})}{\boldsymbol{P}_{\boldsymbol{i}}(\text { cross })} \quad$ Eq. 15

$B E R=0.5 \operatorname{erfc}(\sqrt{2 X S N R} \quad \mathrm{Eq} .16$ 


\section{7) Four-Wave Mixing Crosstalk in SCM Externally Modulated Optical Link}

Four-wave mixing crosstalk is one of the major limiting factors in SCM/WDM optical fiber communications systems that use narrow channel spacing, low chromatic dispersion and high optical channel power. The time-averaged optical power $P_{i j k}(L, \Delta \beta)$ generated through the FWM process for the frequency component fijk is Written in as [22].

$$
P_{i j k}(L)=\frac{1024 \pi^{2}}{n^{4} \lambda^{2} c^{2}}\left(D X_{1111}\right)^{2}\left(\frac{P_{i} P_{j} P_{k}}{A_{\text {eff }}^{2}}\right)\left|\frac{\left\{e^{(i \Delta \beta-\alpha) L}-1\right\}}{\{i \Delta \beta-\alpha\}}\right|^{2}
$$

where $X_{1111}$ is the third-order nonlinear susceptibility, (which is related to $n_{2}$ nonlinear refractive $\left(\mathrm{m}^{2} / W\right)$. The generated wave efficiency $\eta$, with respect to phase mismatch $\Delta \beta L[10,22]$

$$
\eta=\frac{P_{i j k}(L, \Delta \beta)}{P_{i j k}(L, \Delta \beta=0)}=\left|\frac{\left\{e^{(i \Delta \beta-\alpha) L}-1\right\}}{L_{e f f}\{i \Delta \beta-\alpha\}}\right|^{2}=\frac{\alpha^{2}}{\alpha^{2}+\Delta \beta^{2}}\left[1+\frac{4 e^{-\alpha L} \sin ^{2}\left(\frac{\Delta \beta L}{2}\right)}{\left(1-e^{-\alpha L}\right)^{2}}\right]
$$

where $\Delta \beta$ is the propagation constant difference written as

$$
\frac{2 \pi \lambda^{2}}{C} \Delta f_{i k} \Delta_{j k}\left[D+\frac{\lambda^{2} S}{2 C}\left(\Delta f_{i k}+\Delta f_{j k}\right)\right] \quad E q .19
$$

Assuming the equivalent frequency separation $\Delta f=\left(\Delta f_{i k} * \Delta f_{j k}\right)^{1 / 2} \quad E q .20$

$\Delta \beta=\frac{2 \pi \lambda^{2}}{C} \Delta f^{2}\left[D+\frac{\lambda^{2} S}{2 C}(\Delta f)\right] E q .21$

The time-average optical power generated through the FWM process can be modified in terms of generated wave efficiency as $[10,22]$

$$
P_{i j k}(L)=\frac{1024 \pi^{6}}{n^{4} \lambda^{2} c^{2}}\left(D X_{1111}\right)^{2}\left(\frac{P_{i} P_{j} P_{k}}{A_{e f f}^{2}}\right) L_{e f f}^{2} \eta \quad E q .22
$$

Using $D=6$ (none of frequencies are the same). 


\section{8) Dual Parallel Linearized External Modulators}

The basic configuration of optical dual parallel linearization technique is shown below in figure [5].

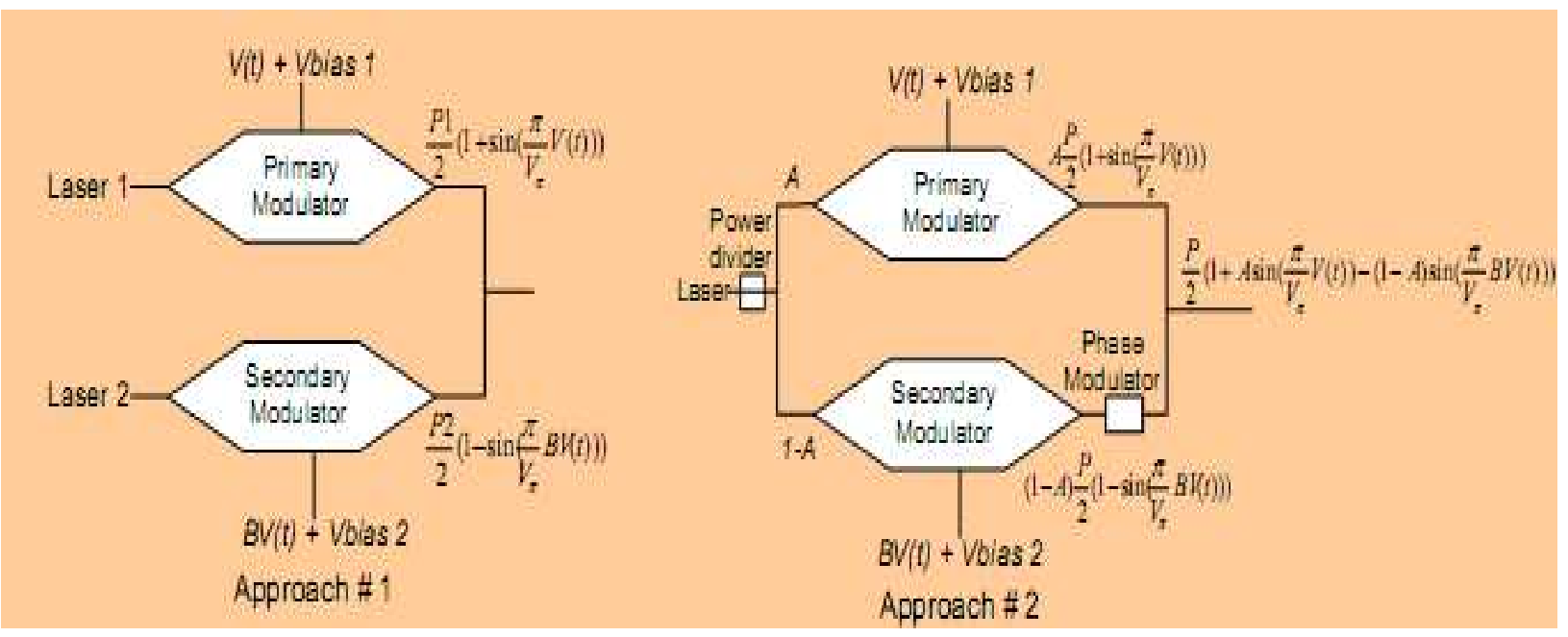

Figure 5: Dual parallel MZ modulator in [6].

By providing less optical power and higher RF drive power to the secondary modulator, the secondary modulator has higher OMI and greater distortion. By providing more optical power to the primary $M Z$ modulator, the third-order distortion products created in the secondary modulator can be made to cancel the distortion products from the primary modulator with a small cancellation of the fundamental signal, result with MATLAB in fig [14-16].

$$
\begin{aligned}
\frac{P_{o u t}}{P i n}=A \cos ^{2}\left[\frac{\frac{\pi V(t)}{V_{\pi}}-\frac{\pi}{2}}{2}\right]+(1-A) \cos ^{2}\left[\frac{B \frac{\pi V(t)}{V_{\pi}}-\frac{3 \pi}{2}}{2}\right] \\
=\frac{1}{2}\left(1+A \sin \frac{\pi V(t)}{V_{\pi}}-(1-A) \sin \frac{B \pi V(t)}{V_{\pi}}\right)
\end{aligned}
$$

where $A$ is the splitting ratio of the input power divider and $B$ is the ratio of $R F$ drive power. Assuming $V(t)$ is a multi-sinusoidal signal, using trigonometric identities and Bessel functions, the amplitude of the fundamental carrier with frequency $\omega_{k}$ can be expressed as [10]:

$$
\frac{P_{\omega k}}{P_{i n}}=A J_{1}\left(\frac{\pi A}{V_{\pi}}\right) J_{O}\left(\frac{\pi A}{V_{\pi}}\right)^{N-1}-(1-A) J_{1}\left(B \frac{\pi A}{V_{\pi}}\right) J_{O}\left(B \frac{\pi A}{V_{\pi}}\right)^{N-1} E q .24
$$


And the amplitude of the third-order intermodulation component of the frequency $\omega_{i}+\omega_{j}+\omega_{k}$ can be expressed as

$$
\frac{P_{\omega_{i}+\omega_{j}+\omega_{k}}}{P_{i n}}=A J_{1}\left(\frac{\pi A}{V_{\pi}}\right)^{3} J_{o}\left(\frac{\pi A}{V_{\pi}}\right)^{N-3}-(1-A) J_{1}\left(B \frac{\pi A}{V_{\pi}}\right)^{3} J_{o}\left(B \frac{\pi A}{V_{\pi}}\right)^{N-3}
$$

The third-order intermodulation product can be cancelled when

$$
A=\frac{J_{1}\left(B \frac{\pi A}{V_{\pi}}\right)^{3} J_{o}\left(B \frac{\pi A}{V_{\pi}}\right)^{N-3}}{J_{1}\left(\frac{\pi A}{V_{\pi}}\right)^{3} J_{o}\left(\frac{\pi A}{V_{\pi}}\right)^{N-3}+J_{1}\left(B \frac{\pi A}{V_{\pi}}\right)^{3} J_{o}\left(B \frac{\pi A}{V_{\pi}}\right)^{N-3}} E q .26
$$

\section{9) Results}

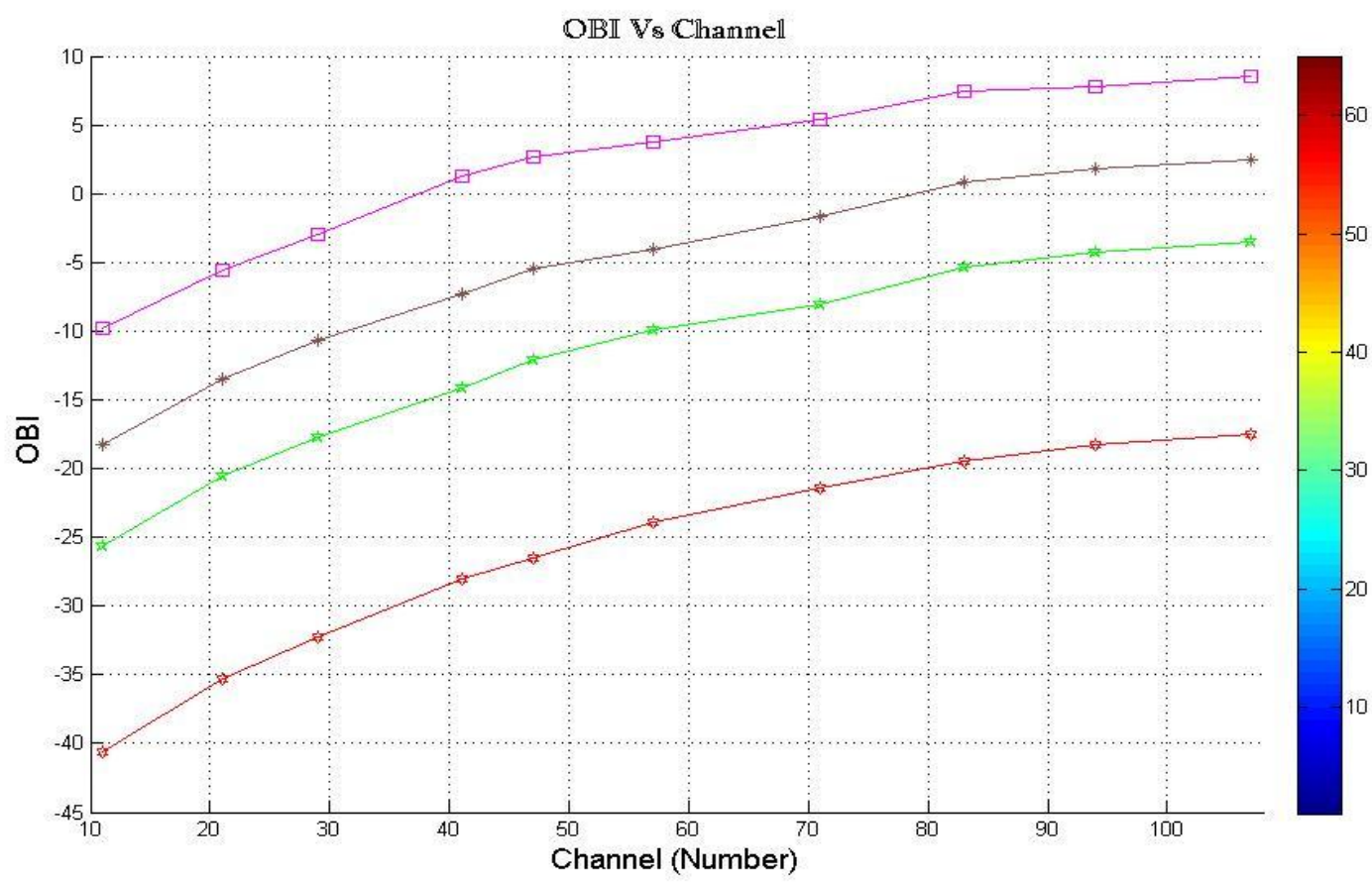

Figure 6: Plot of the optical bit interference $(O B I)$ vs channel number for input power, $P i=1 \mathrm{~dB}, 10 \mathrm{~dB}$ and $20 \mathrm{~dB}$. 


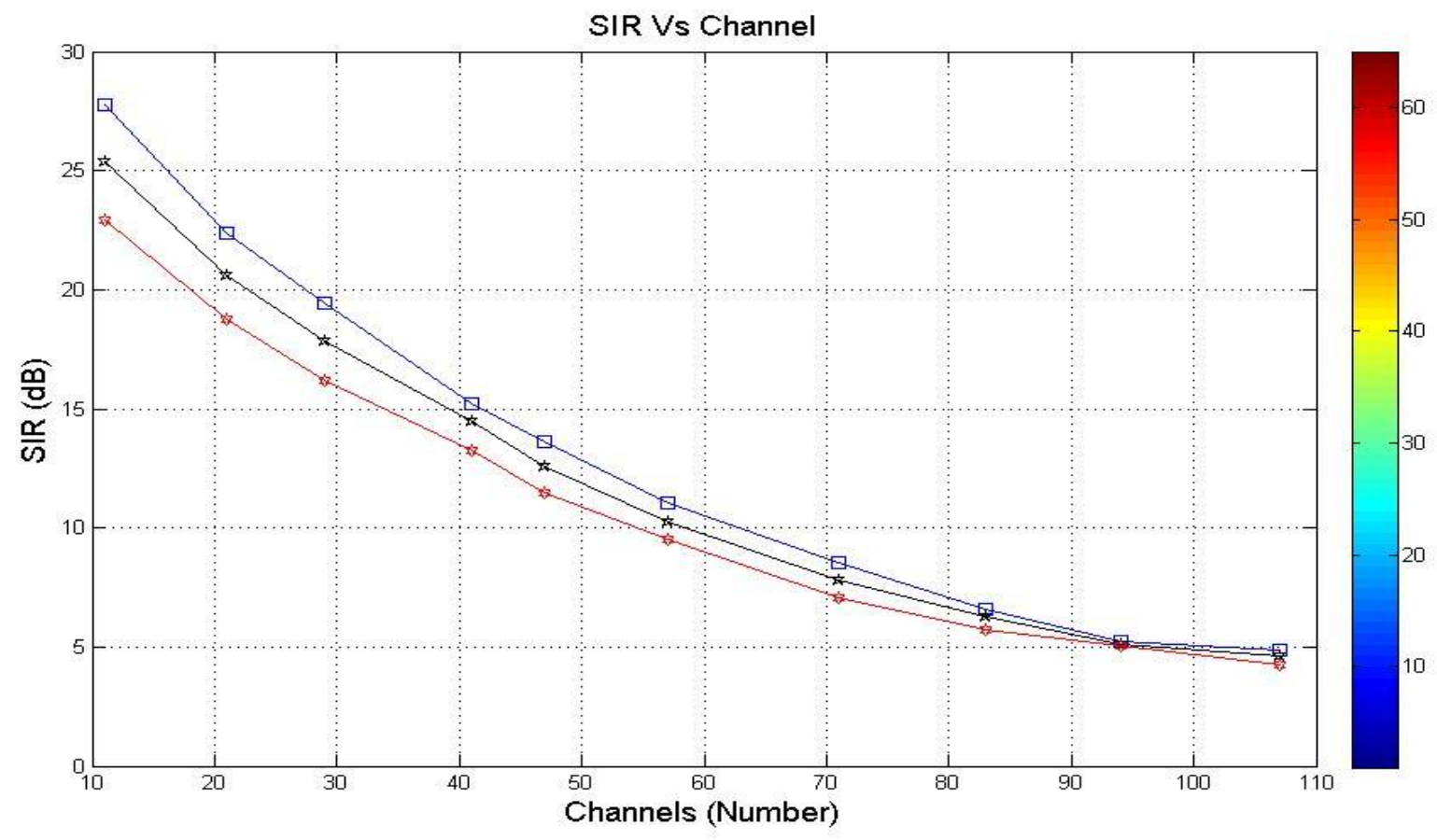

Figure 7: Plot of the signal to interference ratio (SIR) vs channel number for input power, $P i=1 \mathrm{~dB}, 10 \mathrm{~dB}$ and $20 \mathrm{~dB}$.

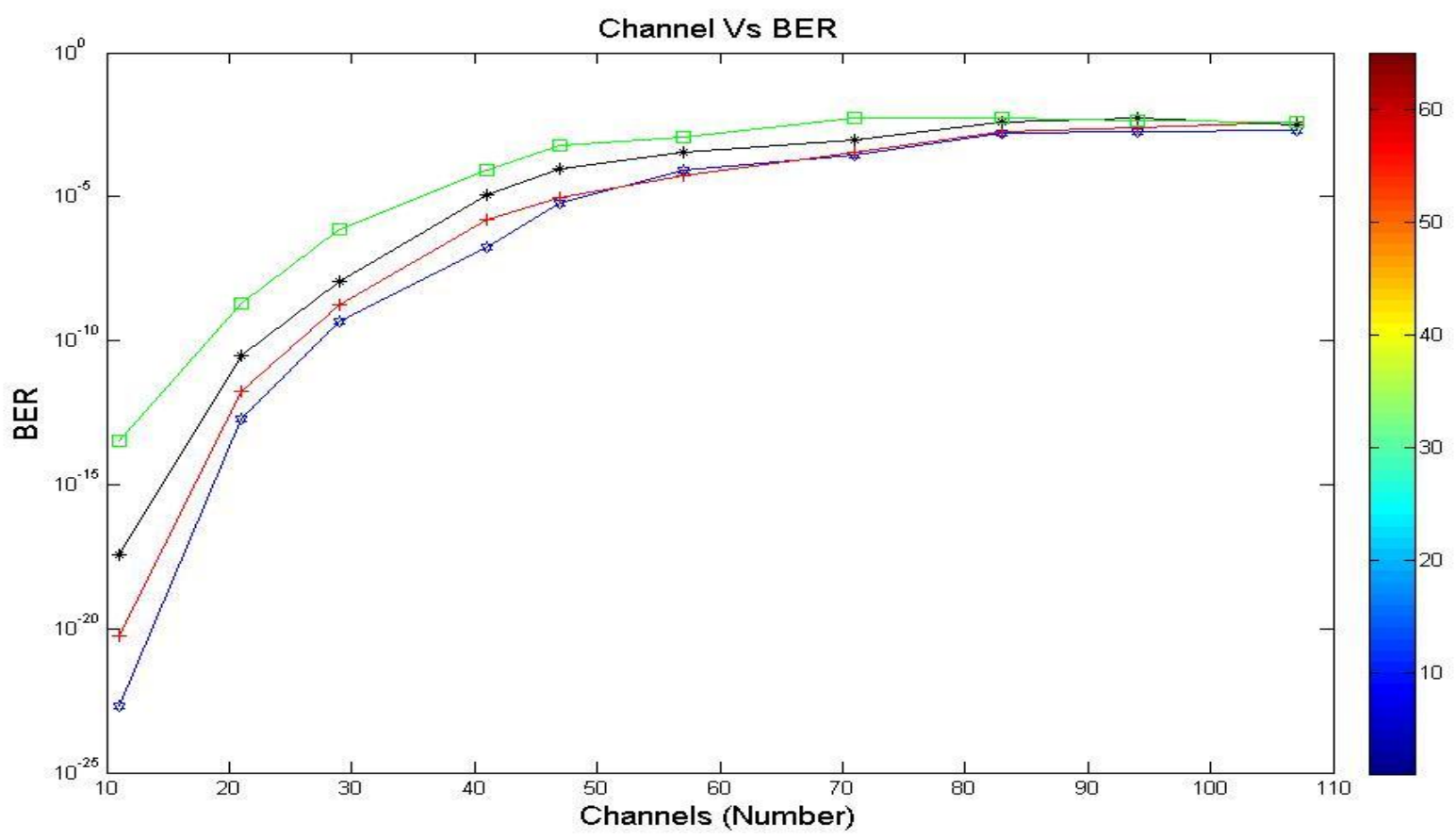

Figure 8: Plot of the bit-error-rate (BER) vs channel number for input power, $\mathrm{Pi}=1 \mathrm{~dB}, 10 \mathrm{~dB}$ and $20 \mathrm{~dB}$. 


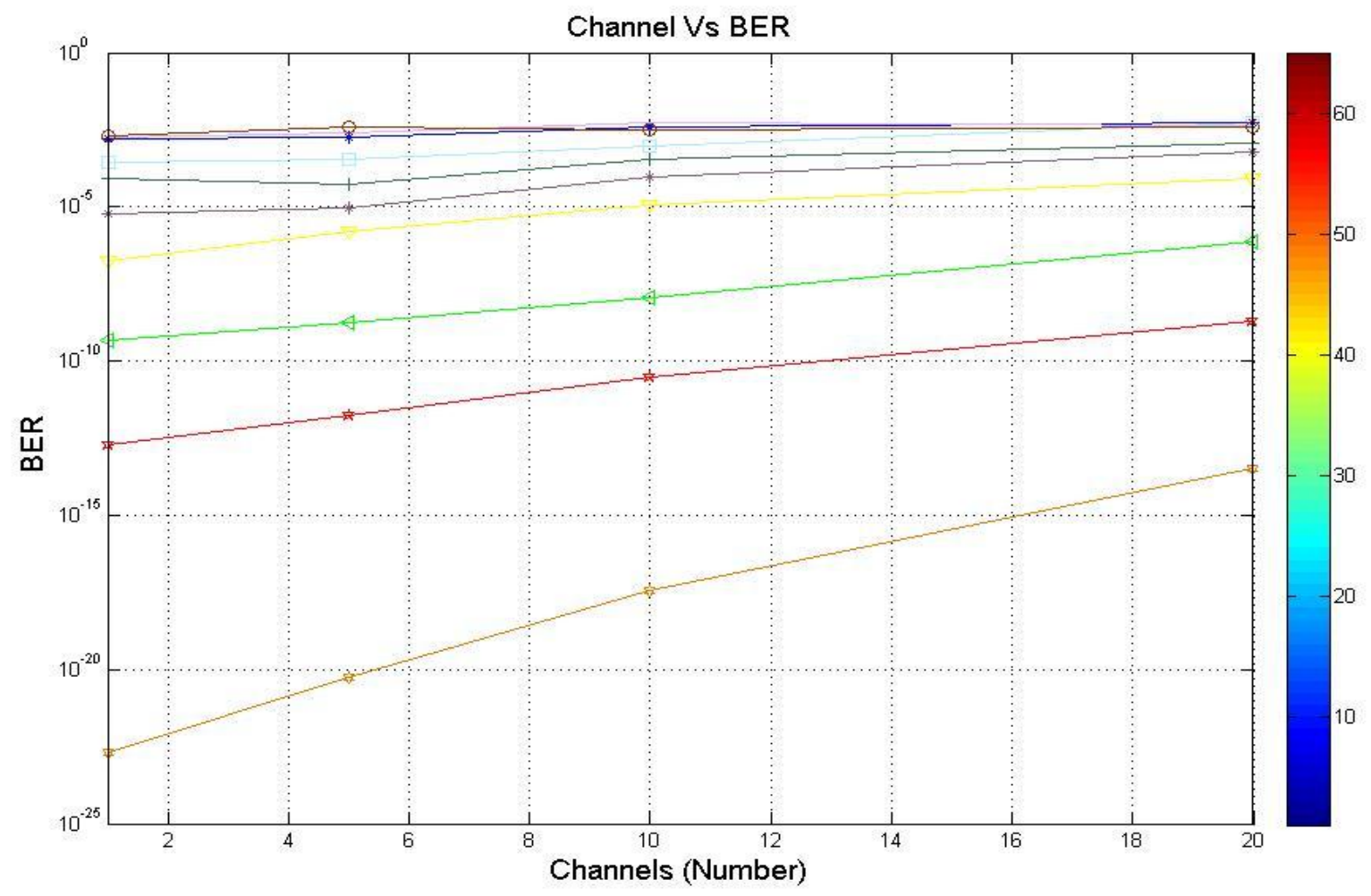

Figure 9: Channel number vs. BER.

\section{0) Discussion}

The number of channels can be increased without significant penalty if the input power is kept low. The number of channels can also be increased if the bandwidth is taken more for more carrier separation.

- Parameters of Design Consideration $[10,16]$

Bandwidth: $890 \mathrm{MHz}-960 \mathrm{MHz}$.

Channel Spacing: $200 \mathrm{KHz}$.

Modulation: QPSK modulation.

Line Coding: NRZ input data.

Interchannel Spacing and number of Channels: $250 \mathrm{KHz}$.

Noise: Noise other than OBI is not considered. 


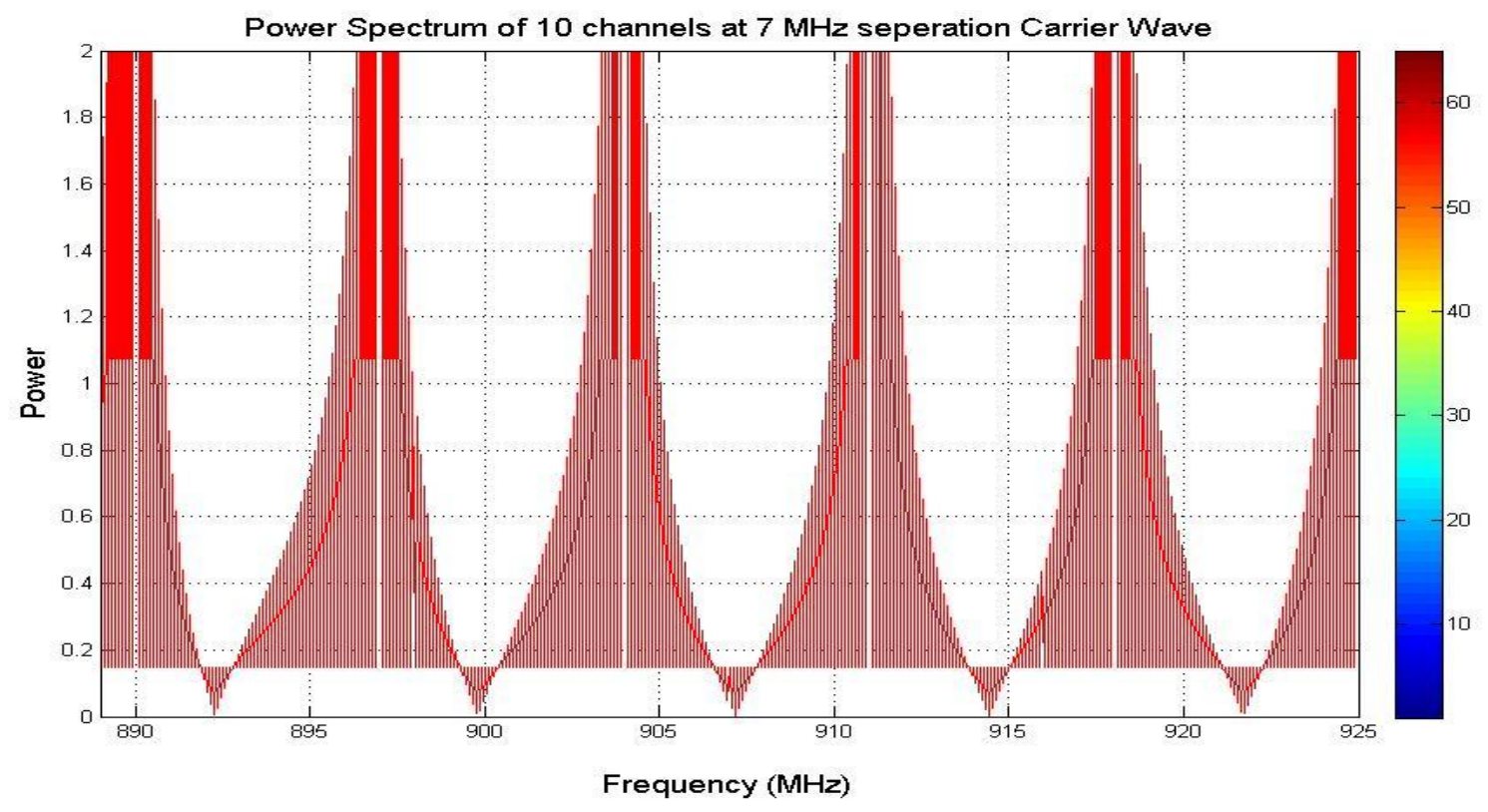

Figure 10: Power spectrum of 10 channels at $7 \mathrm{MHz}$ separation carrier wave.

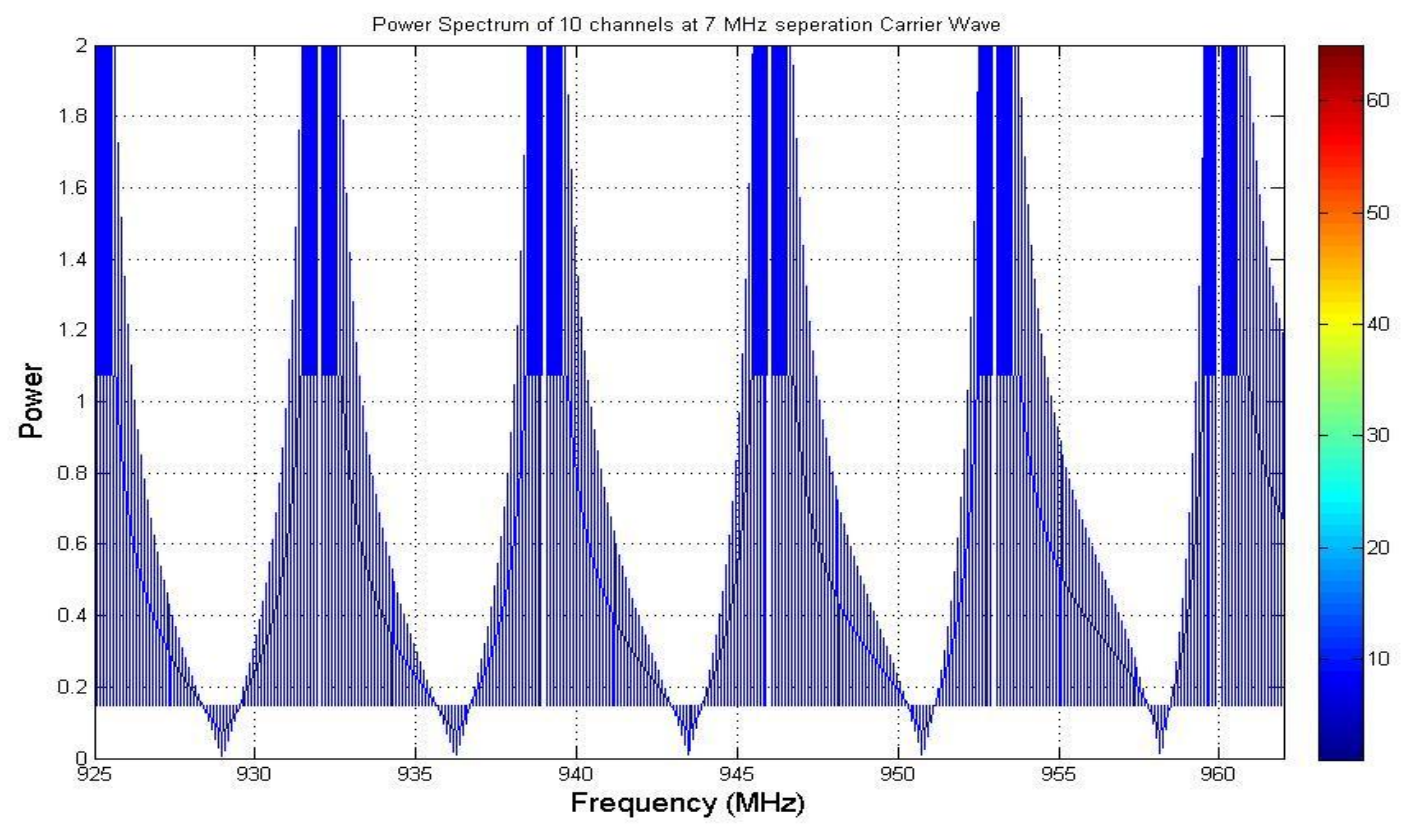

Figure 11: Power Spectrum of 10 channels at $7 \mathrm{MHz}$ separation carrier wave. 


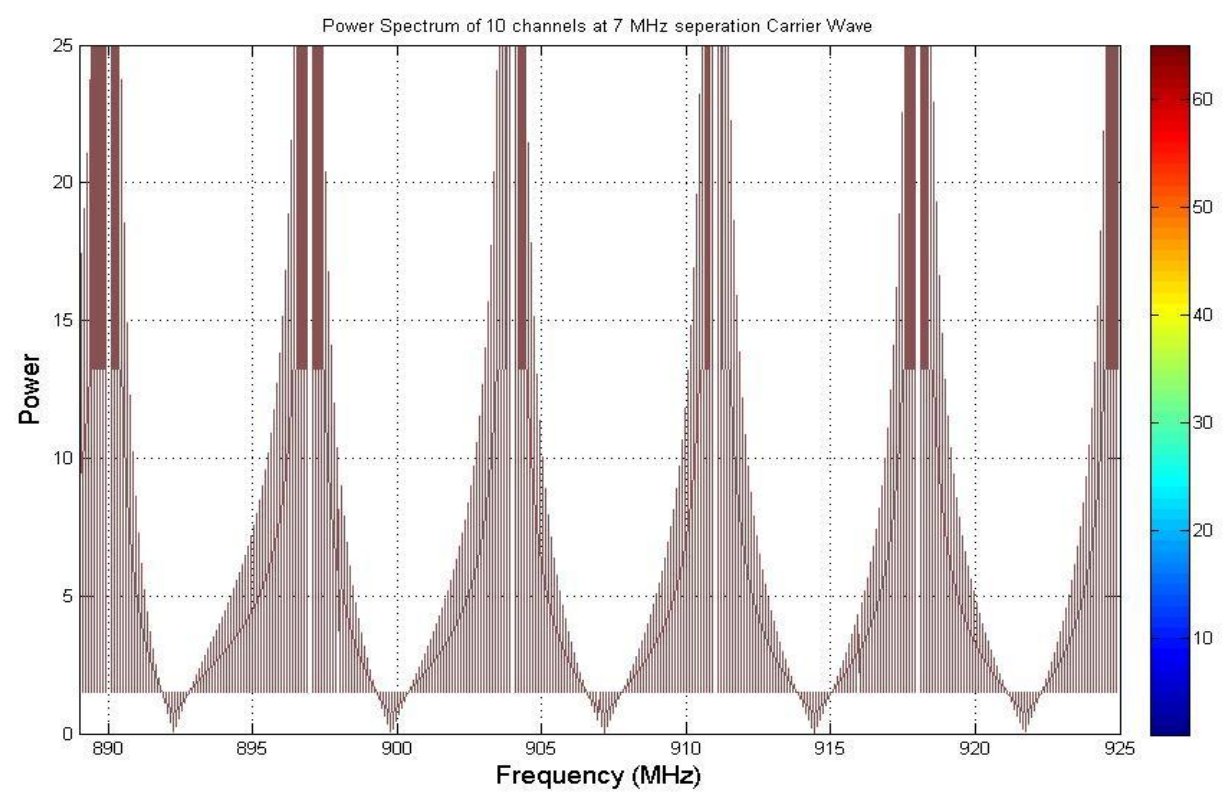

Figure 12: Power spectrum of 10 channels at $7 \mathrm{MHz}$ separation carrier wave.

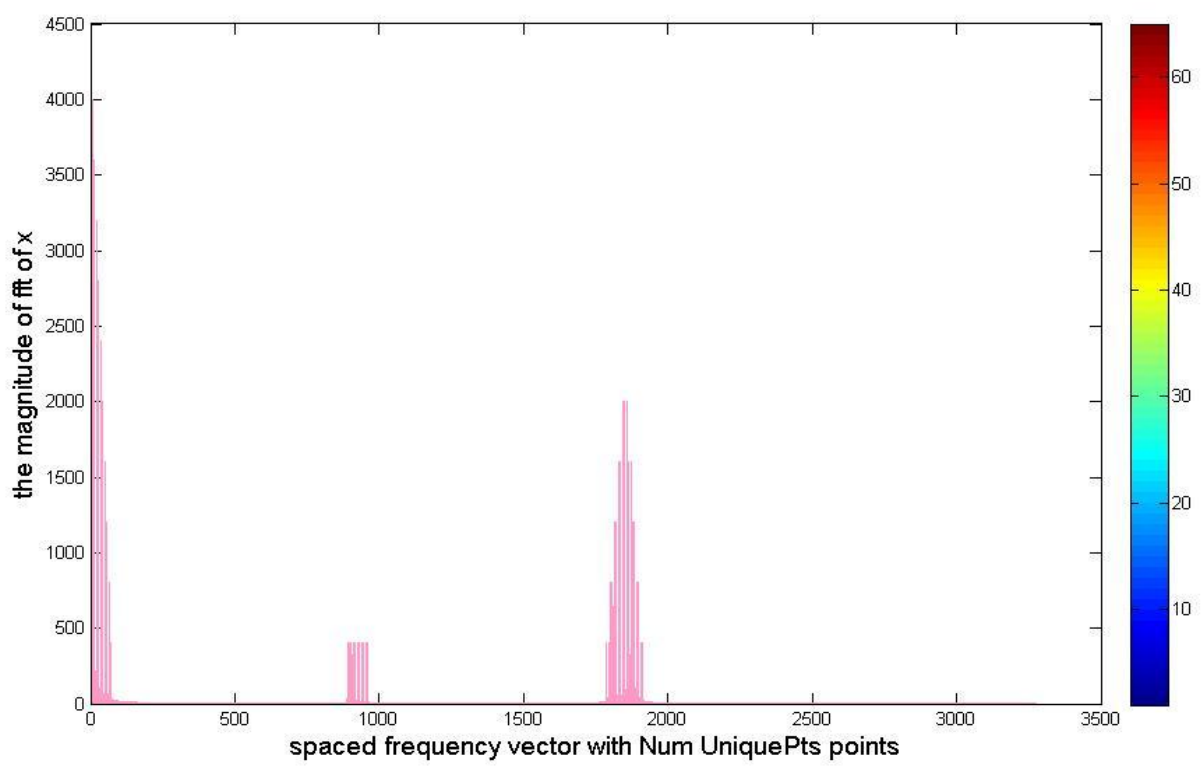

Figure 13: Spaced frequency vector with NumUnique Pts points. And the magnitude of $\mathrm{fft}$ of $x$ and scale the $\mathrm{fft}$ so that it is not a function of the length of $x$. 


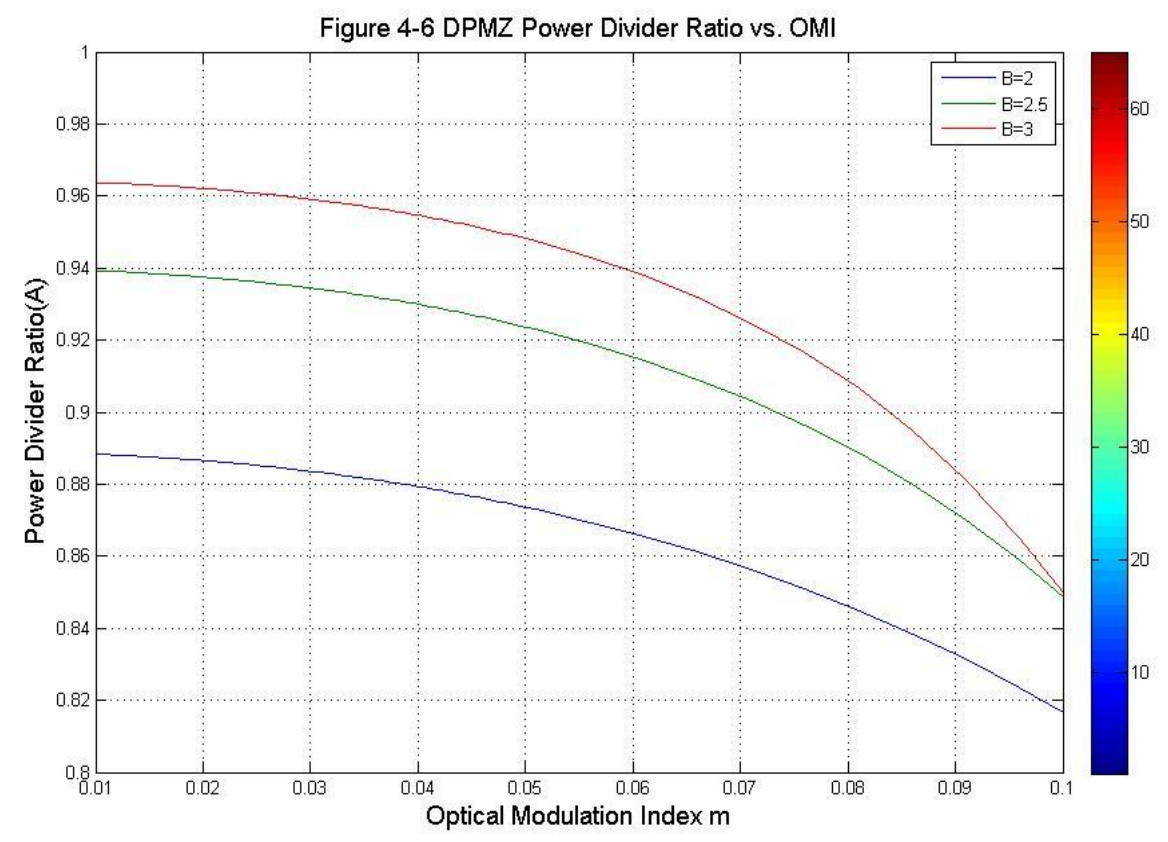

Figure 14: DPMZ power divider ratio vs. OMI (optical modulation index).

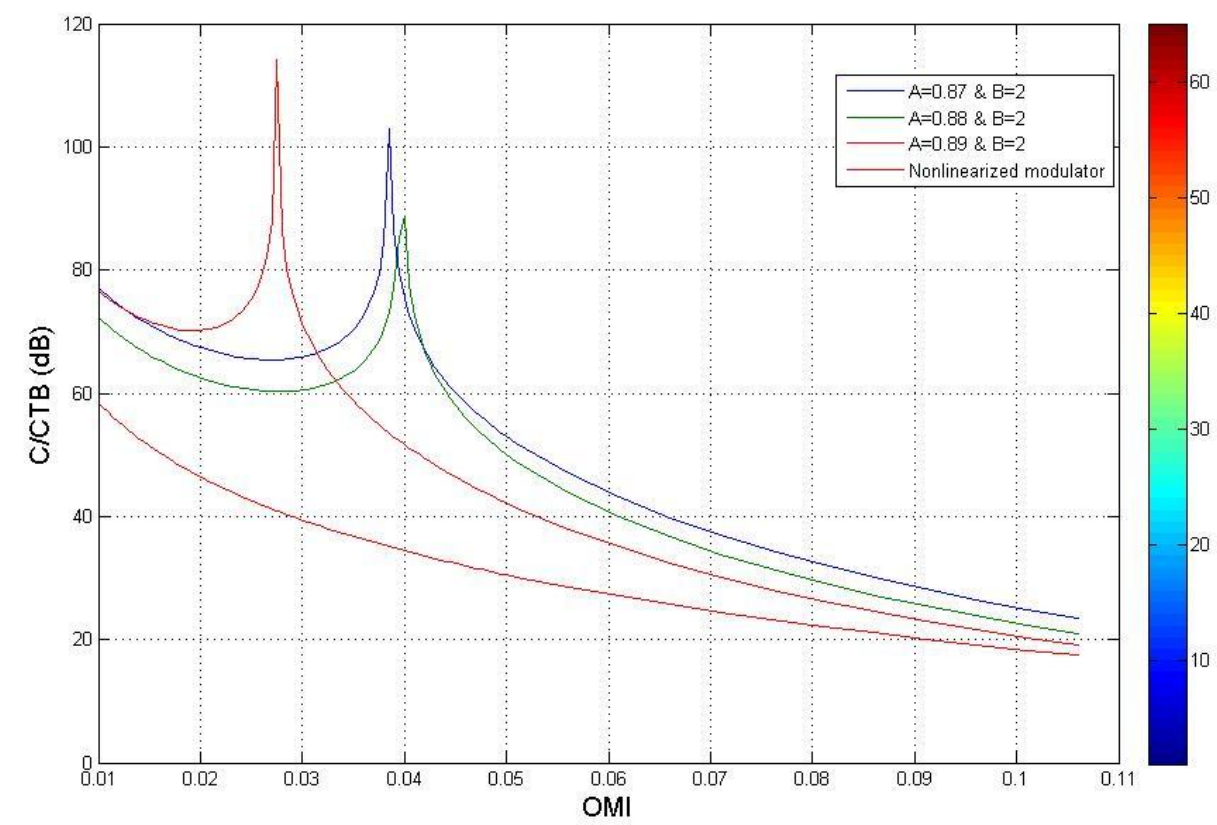

Figure 15: C/CTB performance with $B=2$ and $A=0.87,0.88 \& 0.89$. 


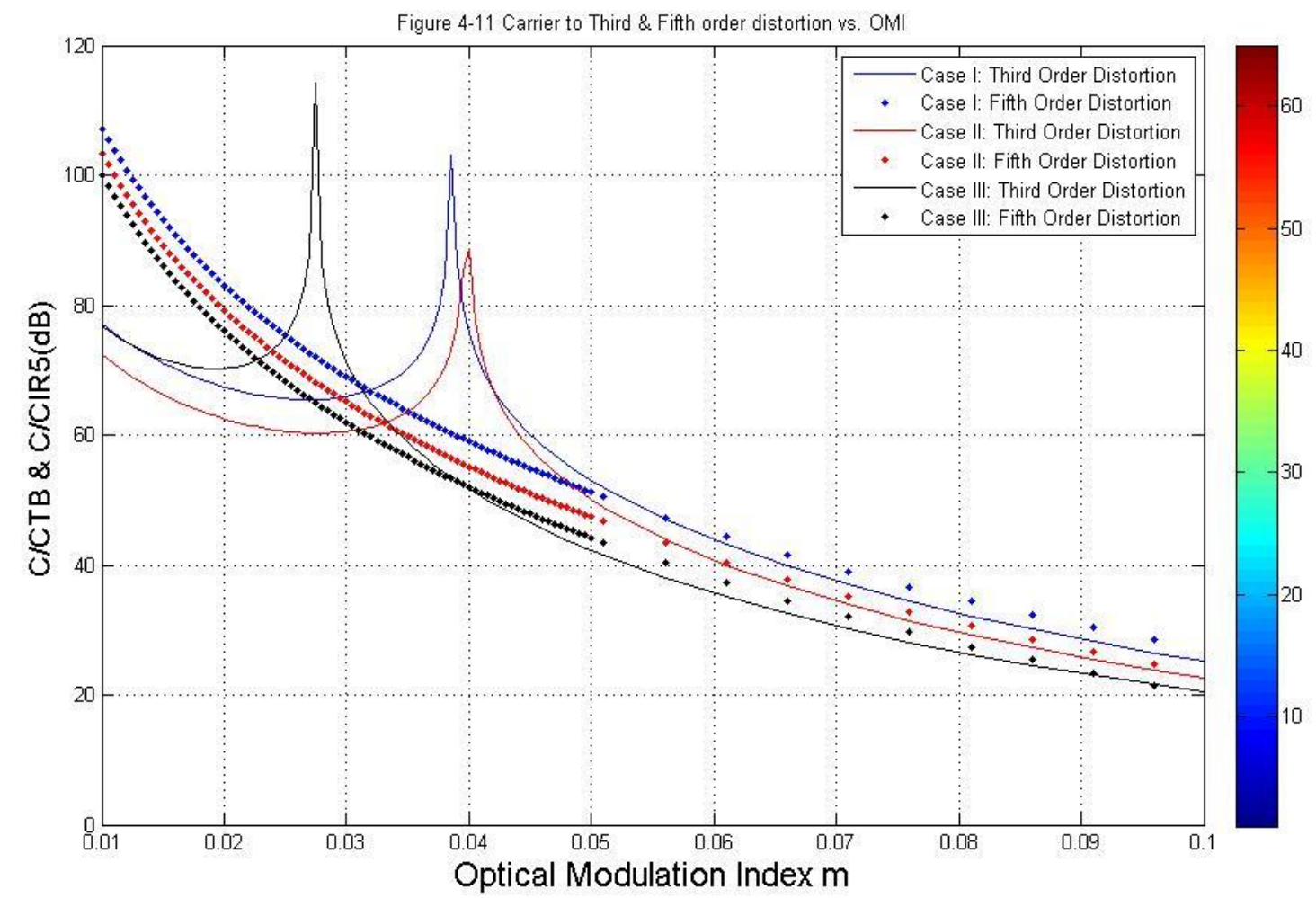

Figure 16: Carrier to third \& fifth order distortion vs. OMI.

\section{1) Source Code}

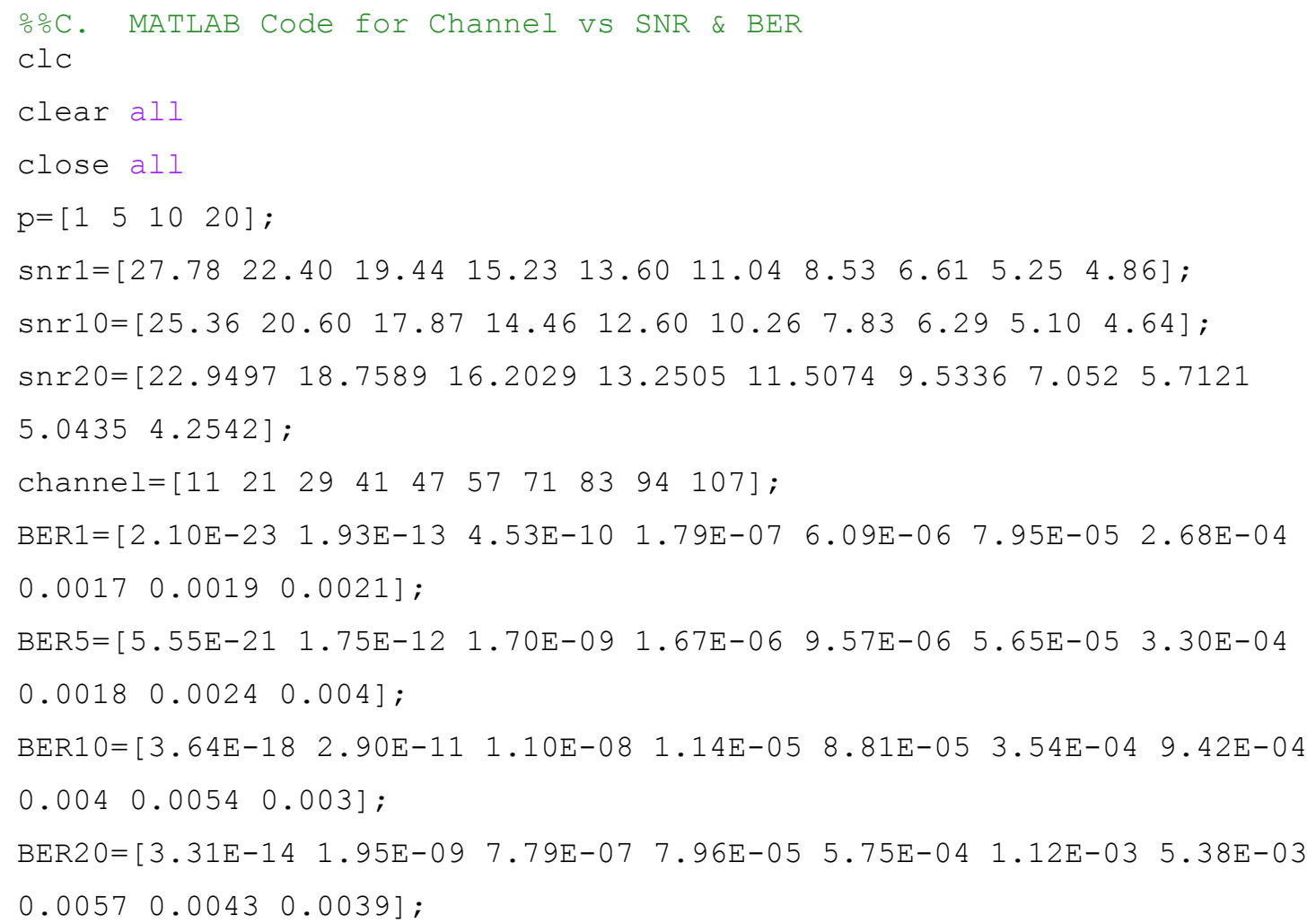




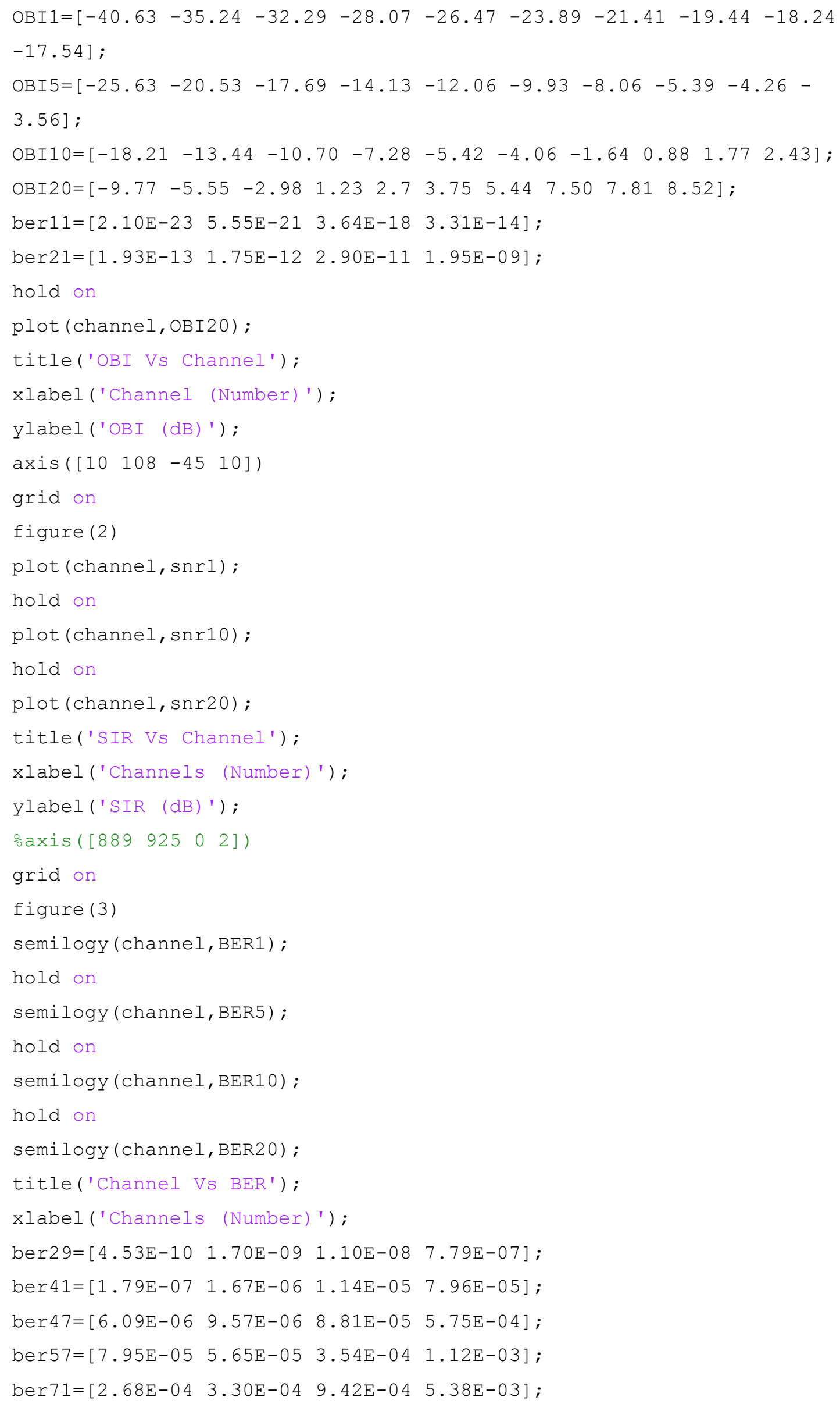




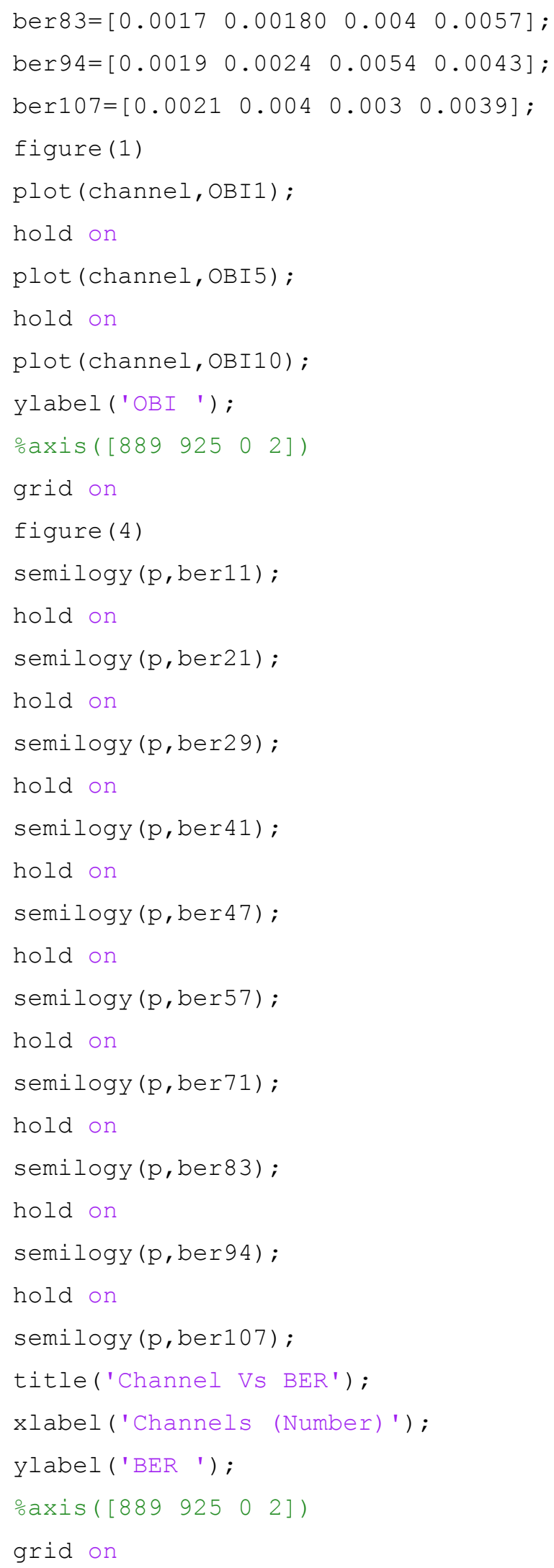




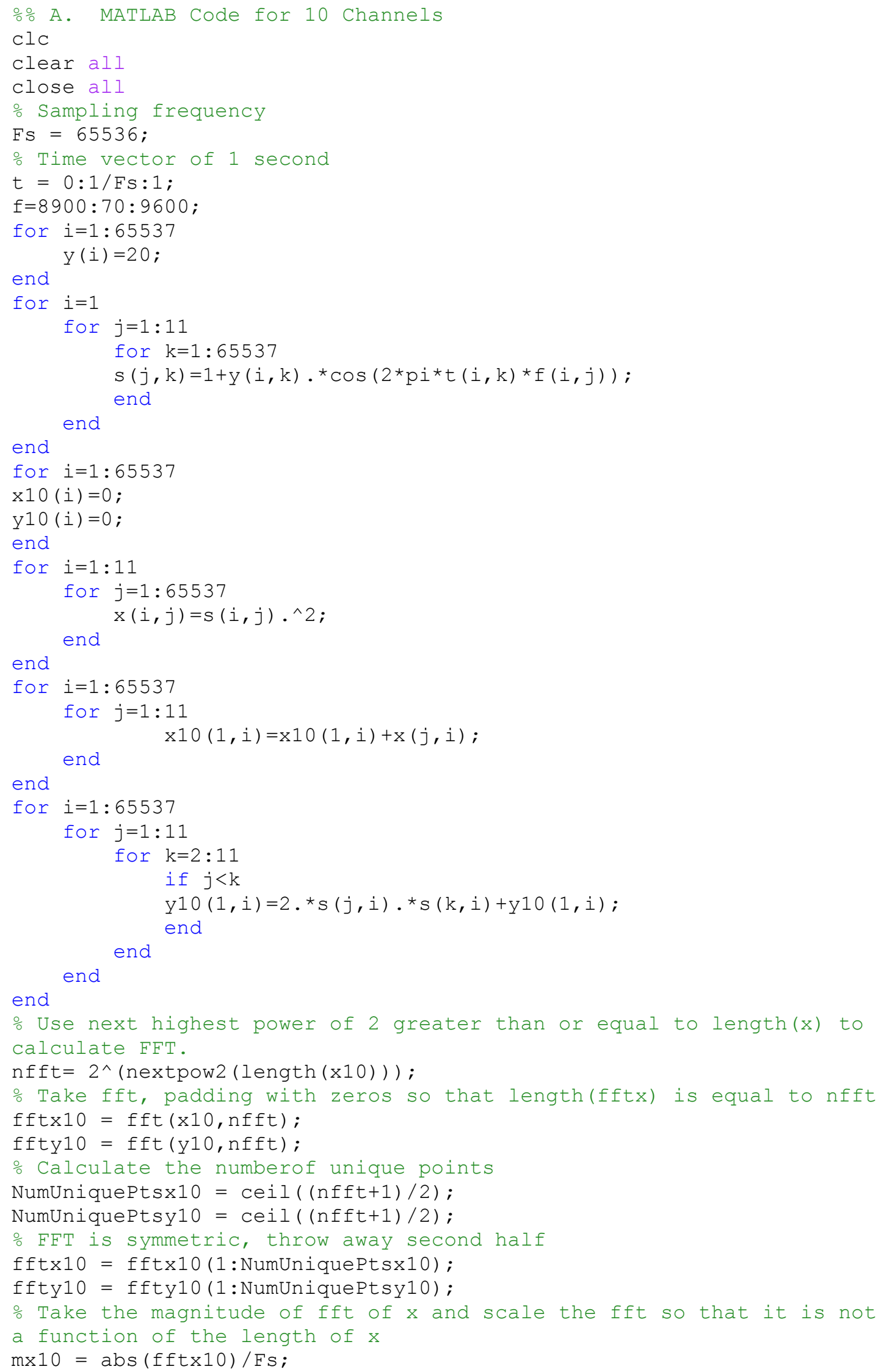




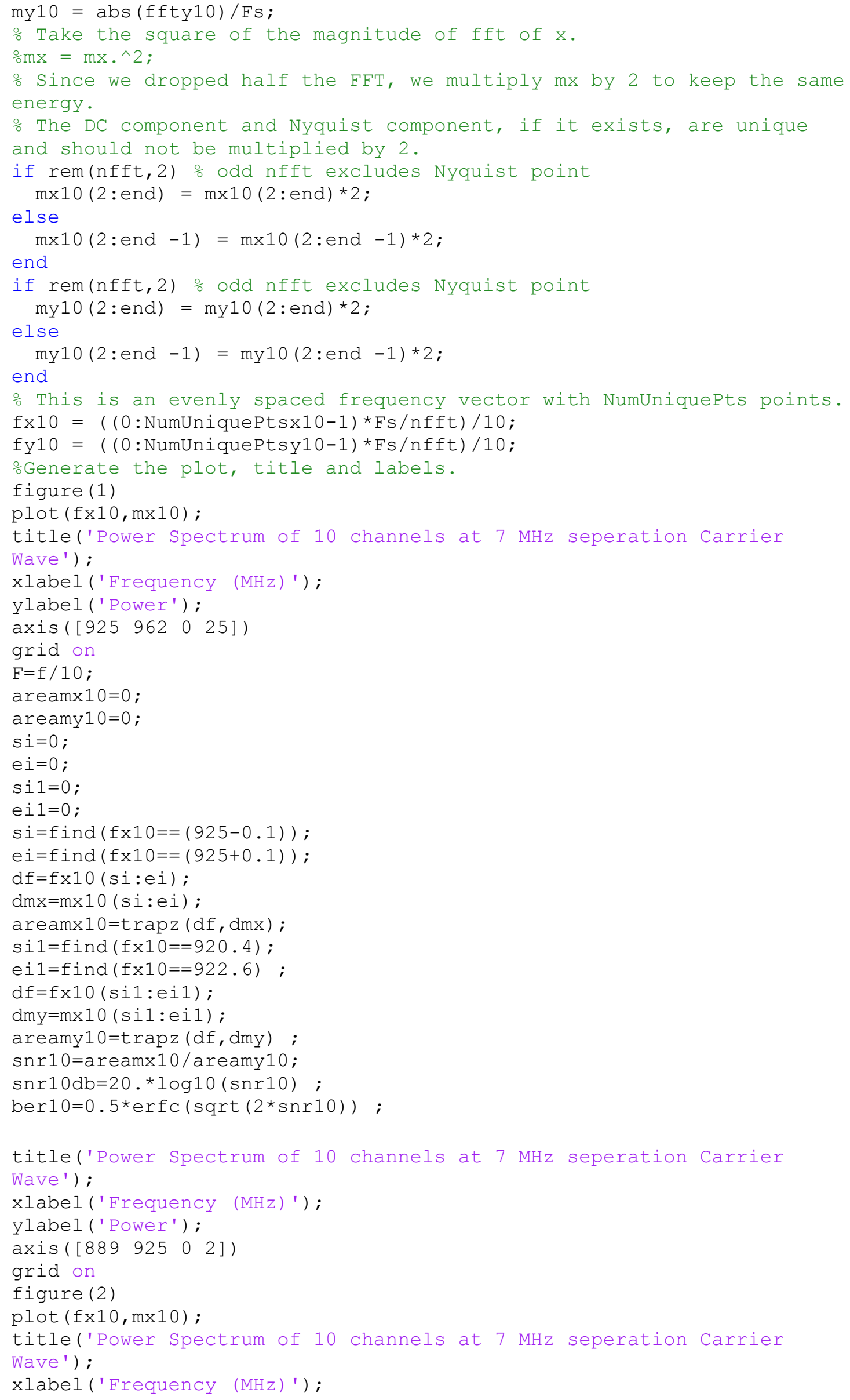




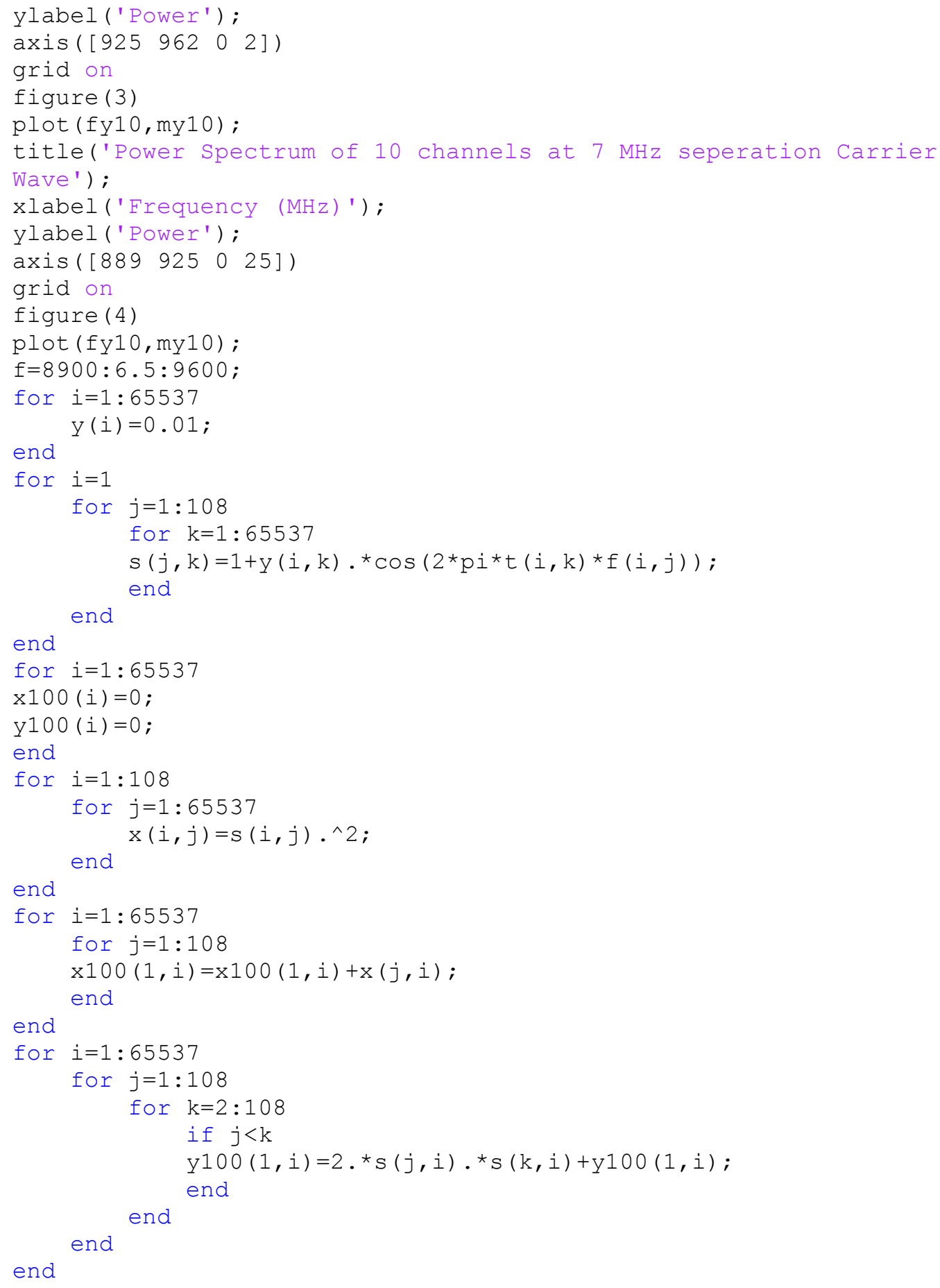




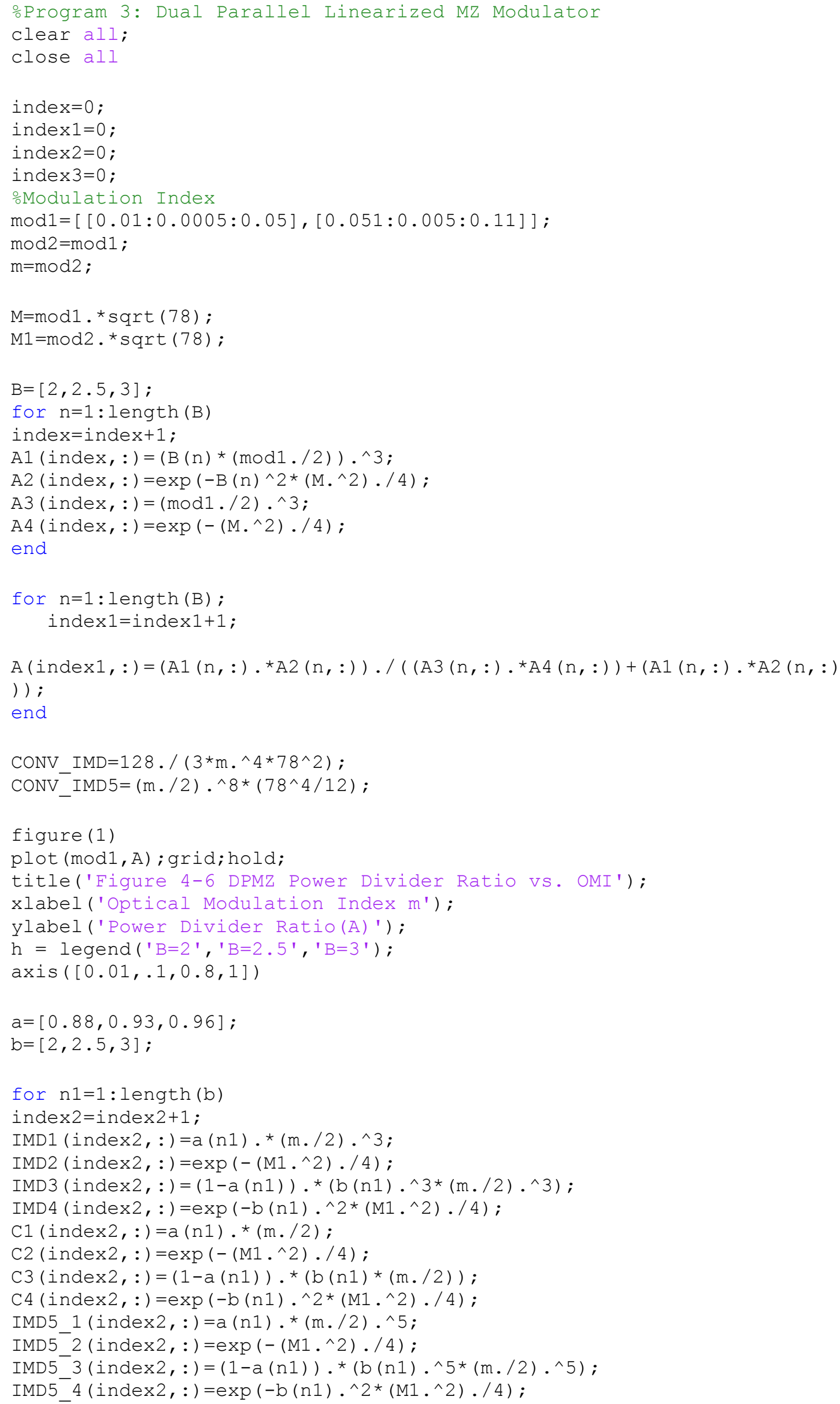




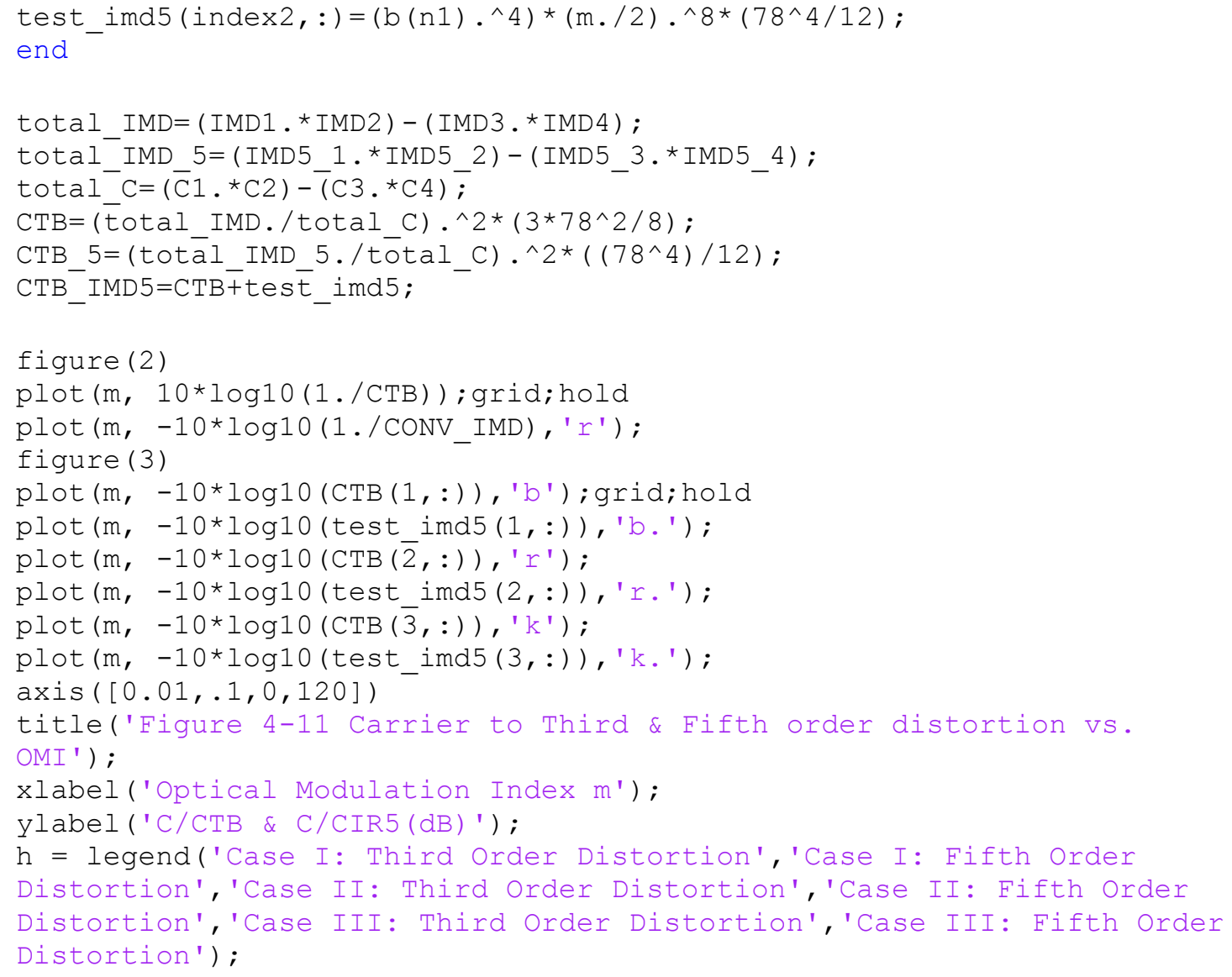




\section{References}

[1] R. Hui, B. Zhu, R. Huang, C. Allen, K. Demarest, D. Richards, "Subcarrier Multiplexing for High-speed Optical Transmission," Journal of Lightwave Technology, vol. 20, no. 3, March 2002.

[2] R. Olshansky, "Optimal Design of Subcarrier Multiplexed lightwave systems employing linearized external modulators," Journal of Lightwave Technology, vol. 10, no.3 March, 1992.

[3] G Keiser, Optical fiber Communication, Boston, 2000.

[4] W. Way, Broadband Hybrid Fiber/Coax Access System Technologies, San Diego, CA: Academic, 1999.

[5] G. Smith, D. Novak, Z. Ahmed, "Overcoming Chromatic-Dispersion Effects in Fiber-Wireless Systems Incorporating External Modulators," IEEE Transactions on Microwave Theory and Techniques, vol. 45, no. 8, August 1997.

[6] J. Brooks, G. Maurer, R. Becker, "Implementation and Evaluation of a Dual Parallel Linearization System for AM-SCM Video Transmission," Journal of Lightwave Technology, vol. 11, no. 1, January 1993.

[7] FCC Standard and Regulations: Section 76.605 "Multichannel Video and Cable Television Service".

[8] J. Chiddix, H. Laor, D. Pangrac, L. Williamson \& R. Wolfe, "AM Video on Fiber in CATV systems: Need and Implementation", IEEE Journal on Selected Areas in Communications, vol. 8, no. 7, September 1990.

[9] S. Bigo, S. Gauchard, A. Bertaina, \& J. Hamaide, "Experimental Investigation of Stimulated Raman Scattering Limitation on WDM Transmission Over Various Types of Fiber Infrastructures," IEEE Photonics Technology Letters, vol. 11, no. 6 June 1999.

[10] M Phillips \& D. Ott, "Crosstalk Due to Optical Fiber Nonlinearities in WDM CATV Lightwave Systems," Journal of Lightwave Technology, vol 17, no. 10, Oct. 1999.

[11] Simon Haykin, Communication Systems (John Wiley \& Sons Inc., 4 Editions.)

[12] Simon Haykin, Digital Communications (Wiley India Edition, 1st Edition.)

[13] Lathi B.P., Modern Digital and Analog Communication Systems (Oxford University Press, 3rd Edition.)

[14] Yang S. \& Yao J.G., Impact of Crosstalk Induced Beat Noise on the size of Semiconductor Laser Amplifier Based Optical Space Switch Structures, IEEE Photonics Technology Letters, Vol. 4. , No. 7, July 1996.

[15] Gyselings Tim, Morthier Geert \& Baets Roel, Crosstalk Analysis of Multiwavelength Optical Cross Connects, Journal of Light wave Technology, Vol. 17, No. 8, August 1999. 
[16] Mizuochi Takashi, Kitayama Tadayoshi, Shimizu Katshuhiro \& Ito Atsuyoshi, Interferometric Crosstalk-Free Optical Add/Drop Multiplexer Using MachZehnder Based Fiber Gratings, Journal of Light wave Technology, Vol 16, No. 2, February 1998.

[17] Phase Modulation in Wikipedia, Available: http://en.wikipedia.org/wiki/phase modulation

[18] Timercon website [online]. Available: http://en.timercon.com/BER/.

[19] Accumulated noise in yahoo answer. Available: http://en.answer.com/topic/accumulated_noise/ (2002) The IEEE website. [Online] Available: http://ieee_xplore.com/topic/ effect_of_crosstalk _on_wdm_mux-demux/

[20] Crosstalk topics. Available: http://www.crosstalkamerica.com/.

[21] Modulation in Wikipedia. Available: http://en.wikipedia.org/wiki/Modulation

[22] N. Shibata, R. Braun, R. Waarts, "Phase Mismatch Dependence of Efficiency of Wave Generation Through Four-Wave Mixing in a Single-Mode Optical Fiber," IEEE Journal of Quantum Electronics, vol QE-23, no. 7, July 1987.

[23] E. E. Elsayed and B. B. Yousif, "Performance evaluation and enhancement of the modified OOK based IM/DD techniques for hybrid fiber/FSO communication over WDM-PON systems," Opt. Quantum Electron., vol. 52, no. 9, 2020, doi: 10.1007/s11082-020-02497-0.

[24] B. B. Yousif, E. E. Elsayed, and M. M. Alzalabani, "Atmospheric turbulence mitigation using spatial mode multiplexing and modified pulse position modulation in hybrid RF/FSO orbital-angular-momentum multiplexed based on MIMO wireless communications system," Opt. Commun., vol. 436, pp. 197-208, 2019, doi: 10.1016/j.optcom.2018.12.034.

[25] A. M. Mbah, J. G. Walker, and A. J. Phillips, "Outage probability of WDM freespace optical systems affected by turbulence-accentuated interchannel crosstalk," IET Optoelectron., vol. 11, no. 3, pp. 91-97, 2017, doi: 10.1049/ietopt.2016.0057.

[26] B. B. Yousif and E. E. Elsayed, "Performance Enhancement of an OrbitalAngular-Momentum-Multiplexed Free-Space Optical Link under Atmospheric Turbulence Effects Using Spatial-Mode Multiplexing and Hybrid Diversity Based on Adaptive MIMO Equalization," IEEE Access, vol. 7, pp. 84401-84412, 2019, doi: 10.1109/ACCESS.2019.2924531.

[27] E. E. Elsayed and B. B. Yousif, "Performance enhancement of hybrid diversity for M-ary modified pulse-position modulation and spatial modulation of MIMO-FSO systems under the atmospheric turbulence effects with geometric spreading," Opt. Quantum Electron., vol. 52, no. 12, 2020, doi: 10.1007/s11082-020-02612-1.

[28] Ebrahim E. Elsayed, Bedir B. Yousif, and Mahmoud M. Alzalabani, "Performance enhancement of the power penalty in DWDM FSO 
communication using DPPM and OOK modulation", Optical and Quantum Electronics, vol. 50 (7), pp. 282, (26 June 2018).

[29] Ansari, N., Zhang, J.: 'Media access control and resource allocation for next generation passive optical networks' (Springer, 2013)

[30] Ebrahim E. Elsayed and Bedir B. Yousif, "Performance enhancement of the average spectral efficiency using an aperture averaging and spatial-coherence diversity based on the modified-PPM modulation for MISO FSO links", Optics Communications, vol. 463, pp. 125463, (15 May 2020).

\section{Compliance with ethical standards}

Conflict of interest: The author declares that there is no conflict of interest regarding the manuscript. The author is responsible for the content and writing of this article. The author declares that he has no known competing financial interests or personal relationships that could have appeared to influence the work reported in this paper. 